\title{
NON-COULOMB PERTURBATIONS INFLUENCE ON BEAM DYNAMICS IN EXTENDED ACCELERATING/FOCUSING CHANNELS
}

\author{
Boris Bondarev, Alexander Durkin \\ Moscow Radiotechnical Institute \\ 113519,Russia,Moscow, Warshawskoeshosse 132,lidos@aha.ru
}

It is generally taken that the main source of particle losses in extended linac channel is the Coulomb field of space charge dominated beam. However there are the non-Coulomb effects concerned with external field perturbations (external perturbations) and their influence on beam dynamic is comparable with the action of space charge.

It is convenient to split external perturbations into two groups: constructive (regular) perturbations caused by distinction of a real structure from an ideal one and perturbations caused by random parameter deviations within given tolerances. In the last case only the probability that the beam size would be no more than given value can be found. In connection with strict requirements for channel transparent and also with the complex procedure for channel retuning and readjustment, the confidence level must be chosen sufficiently large.

The calculation of the accelerating/focusing channel always based on the specific mathematical model involving description of external accelerating and focusing forces.

In this model as a rule, the focusing field linear dependence on transverse coordinates and accelerating field axis distribution is used. The channel based on such models will be called ideal. Of course, there are no unprojected losses in the ideal channel.

Output beam parameters degradation including transverse size and emittance growths is caused by channel and beam parameter perturbations (not always small). The influence of perturbations upon the beam output parameters determines by quantity, which have come to be known as the channel sensitivity. The goals of beam dynamics investigations are:

- knowledge about channel sensitivity - each perturbing factor influences output beam parameters,

- perturbing factors compensation possibilities,

- redundant factor determination guaranteed the beam passing throughout the real channel without losses.

From strategy standpoint, the major goal of the investigation is determination of the generalized parameters whose numerical values permit to judge about channel sensitivity as well as about beam parameter degradation. The confidence level must be sufficient for intolerable beam loss estimation. The cardinal problem is to choose the channel with minimum sensitivity in order to minimize the beam losses during the process of accelerating.
Mathematical foundation and the main treatments are given in the works [1-6] as well as the tolerance estimation for various types of focusing and accelerating channel. Below we will investigate the parameters determining the sensitivity of long channel to external perturbations.

Let us consider as example motion of particles in focusing channel with random errors in focusing field gradients

$$
x^{\prime \prime}+\widetilde{G}(z) \cdot x=0
$$

Let us consider $\tilde{G}(z)$ as $\widetilde{G}(z)=G(z) \cdot(1+\alpha(z))$, where $G(z)$ is non-perturbed field gradient, $\alpha(z)$ is function for perturbation description.

As is shown in above cited works we need to do transformation of variables $x, d x / d z$ to phase variables $r, \eta$. $r^{2}$ is quadric in $x, d x / d z$, describing the ellipses matched with the ideal periodical channel. $\eta$ is an addition due to external perturbations. We obtain as a result the main equation in form

$$
\left\{\begin{array}{l}
r^{\prime}=r \cdot \alpha \cdot G \cdot \rho^{2} \cdot \sin ^{2}(\mu z+\eta+\psi) \\
\eta^{\prime}=\alpha \cdot G \cdot \rho^{2} \cdot \cos ^{2}(\mu z+\eta+\psi)
\end{array}\right.
$$

Here $\mu$ is phase advance, $\rho$ is the envelope of beam with the emittance of unity in the ideal channel.

The right part of the second equation does not depend on $r$, so it can be solved separately. If $\eta(z)$ is solution of the second equation, then solution of the first equation has the following form

$$
\frac{r(z)}{r_{0}}=\theta(z)=\exp \left(\int_{0}^{z} \alpha G \rho^{2} \sin (\mu t+\eta+\psi) \cos (\mu t+\eta+\psi) d t\right)=\exp (F(z))
$$

It can be seen from obtained relation that distribution of random value $\theta(z)$ is determined by distribution of random value $F(z)$.

If focusing channel contains $N$ periods, then $F(z)$ can be presented as sum of statistical independent components adequate to corresponding periods $L_{k}$

$$
\begin{aligned}
F(N)=\sum_{k=1}^{N} F_{k} & =\sum_{k=1}^{N} \int_{L_{k}} \alpha G \rho^{2} \sin (\mu t+\eta+\psi) \cos (\mu t+\eta+\psi) d t \\
& \text { Using approximate solution of the second }
\end{aligned}
$$
equation

$$
\begin{aligned}
& \eta(N)=\varphi_{0}+\int_{0}^{N} \alpha G \rho^{2} \cos ^{2}\left(\mu t+\varphi_{0}+\psi\right) d t= \\
& \varphi_{0}+\sum_{k=1}^{N} \eta_{k}=\varphi_{0}+\sum_{k=1}^{N} \int_{L_{k}} \alpha G \rho^{2} \cos ^{2}\left(\mu t+\varphi_{0}+\psi\right) d t
\end{aligned}
$$

we obtain with an accuracy of $\alpha^{3}$ 
$F_{k}=\int_{L_{k}} \alpha G \rho^{2}\left(0.5 \sin 2\left(\mu t+\varphi_{0}+\psi\right)-\cos 2\left(\mu t+\varphi_{0}+\psi\right) \cdot \sum_{i=1}^{k} \eta_{k}\right) d t$

Suppose $\alpha(z)$ is piecewise constant function that is equal the relative error of field gradient inside any element of channel. In this case integral is calculated only along the part of period, containing perturbation. If $\sigma^{2}$ is mean square value of field gradient tolerance, then using statistical independence of errors for different focusing periods and averaged over oscillations we obtain relations for the mean value and dispersion for $F(N)$

$$
M[F(N)]=D[F(N)]=\frac{\sigma^{2}}{8} \sum_{k=1}^{N}\left(\overline{G \rho^{2}} \mid\right)_{k}{ }^{2}=\frac{\sigma^{2}}{8} \sum_{k=1}^{N} S_{k}
$$

As shown in [4] if $\sigma^{2}$ is mean square value of focusing field gradient relative error then the probability that effective emittance growth would be no more than $x$ is determined by function $P(x)=1-e^{-(\ln x)^{2} / \Delta^{2}}$, where $\Delta^{2}=\frac{\sigma^{2}}{4} \sum_{k=1}^{N} S_{k}$. If $\sigma^{2}$ is error of axis transverse displacement then the probability that center of output beam displacement would be no more than $x$ is determined by function $P(x)=1-e^{-x^{2} / \Delta^{2}}$, where $\Delta^{2}=\sigma^{2} \sum_{k=1}^{N} S_{k}$. We can give the definition of $S_{k}$ as a sensitivity of period numbered $\mathrm{k}$. Then total sensitivity of channel will be sum of period sensitivities $S=\sum_{k=1}^{N} S_{k}$.

The regions of stability diagrams for RFQ and some types of quadrupole channels are shown in Fig. 1-4. In this regions the isolines of transverse oscillation frequency $\mu$ and sensitivity $S={\overline{\mid Q \rho^{2}}}^{2}$ are plotted. We use traditional coordinates: focusing parameter $B=\frac{e U}{m_{0} c^{2}} \kappa\left(\frac{\lambda}{a}\right)^{2}$ and defocusing parameter $A=\frac{\pi^{2} e U \sigma}{4 W_{s}}\left|\sin \varphi_{s}\right|$ for RFQ diagram. For quadrupole channels we use focusing parameter $Q=\frac{e G L^{2}}{m_{0} c \beta \gamma}$, where $G$ - focusing field gradient, $L$ - length of focusing period, and defocusing parameter $A=\frac{\pi e E_{m} L^{2}}{m_{0} c^{2}(\beta \gamma)^{3} \lambda}\left|\sin \varphi_{s}\right|$, where $E_{m}$ - accelerating wave amplitude, $\lambda$ - RF field wave length. In figures $1-4 \mu$-isolines are corresponded to values (from bottom to top) $\mu=0, \mu=\pi / 3, \mu=\pi / 2, \mu=$ $2 \pi / 3, \mu=\pi$.

As is seen from these diagrams even within the boundaries of operating region of stability diagram the channel sensitivity can be varied two times by value. So for choice and calculations parameters of long linac channel the reported results would be taken into account necessary.

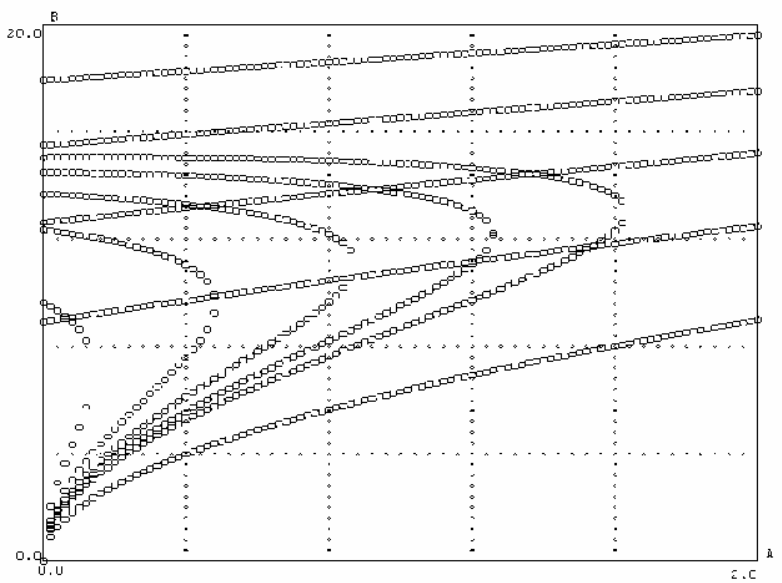

Fig.1. Sensitivity $\mathrm{S}$ of RFQ channel. $1-40,2$ - 50, 3 - 60, 4 - 70, 5- 80 (from left to right)

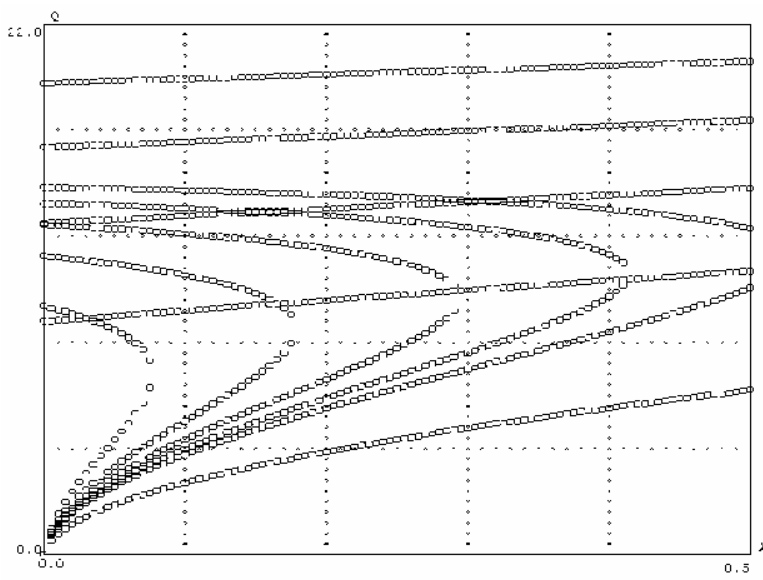

Fig.2. Sensitivity S of FODO channel. 1 - 30, 2 - 35, 3 - 40, 4 - 45, 5- 60 (from left to right; $\mathrm{Gap} / \mathrm{Period}=0.5$ )

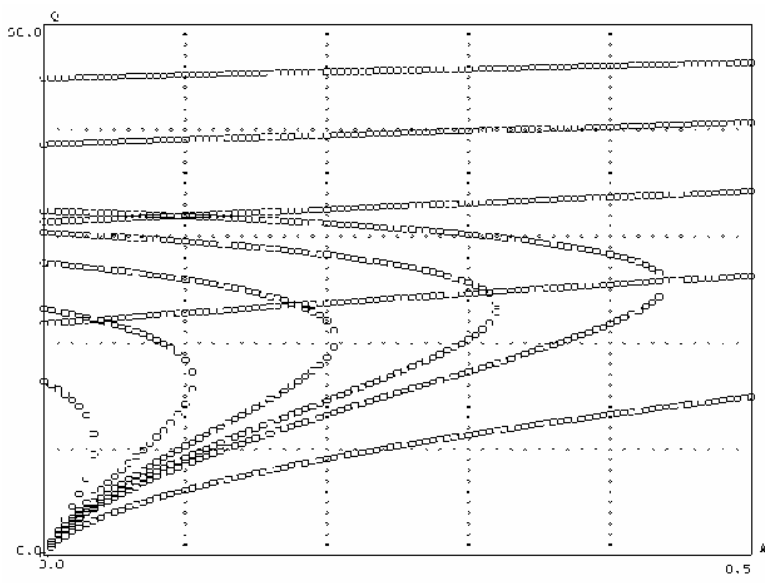

Fig.3. Sensitivity S of FDO channel. 1 - 70, 2 - 85, 3 - 100, 4-115, 5- 130 (from left to right; Gap/Period $=0.8$ ) 


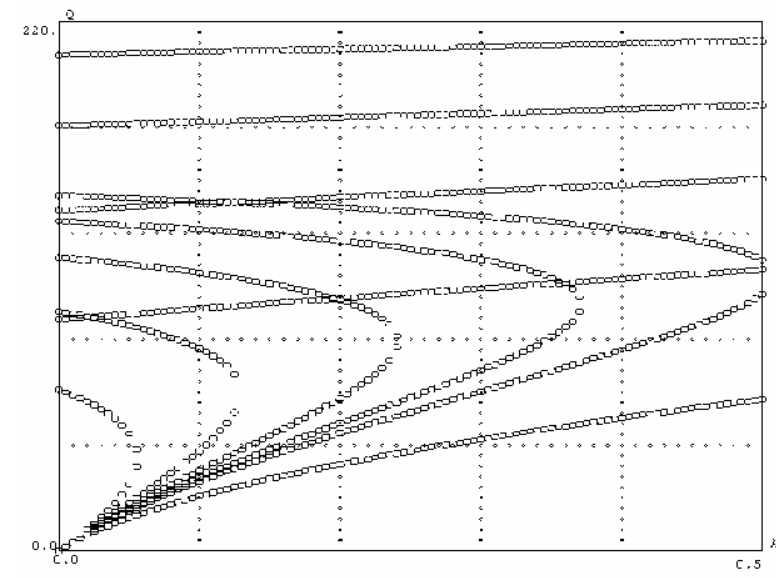

Fig.4. Sensitivity S of FDO channel.

1 - 100, 2 - 130, 3 - 160, 4 - 190, 5- 220

(from left to right; Gap/Period $=0.9$ )

\section{REFERENCES}

[1] B.I.Bondarev, A.P.Durkin, B.P.Murin, L.Yu.Soloviev. Random Perturbation of the Transverse Motion of
Protons in a Linear Accelerator and Their Correction, Particle Accelerators, 1974, Vol.6, N 1, pp.27-40.

[2] A.P.Durkin. The Investigation of Transverse Particles Motion in Linac by the Perturbation Theory Techniques, Dr. Ph. Thesises, Russia, Moscow, 1978 (in Russian).

[3] B.I.Bondarev, A.P.Durkin, L.Yu.Soloviev. Influence of Focusing Fields Errors on the Particles Motion in Linac. Journal of Technical Physics, 1979, v.49, No 8, p.1662-1672. (in Russian)

[4] B.P.Murin, B.I.Bondarev, A.P.Durkin An Approach to the Design of a Robust Focusing Channel. Proceedings of 1992 Linear Accelerator Conference, v.2, pp.731-733. (1992, August 24-28, Ottawa, Ontario, Canada).

[5] B.P.Murin, B.I. Bondarev, A.P.Durkin, L.G.Lomize, L.Yu.Soloviev. Some Aspects of Simultaneous Acceleration of Proton and $\mathrm{H}^{-}$Beams in a Linac, Particle Accelerators, 1975, v.6, No 3, p.133-139.

[6] B.I.Bondarev, A.P.Durkin, B.P.Murin. Effective Emittance Growth. Contract 9-XG3-5167H-1 between LANL and MRTI, Phase 1, Moscow 1993. 